\title{
Podcast on B Cell-Targeting Therapies and Other Multiple Sclerosis Concerns During COVID-19
}

\author{
Robert K. Shin · Pavle Repovic · Joseph R. Berger
}

Received: November 8, 2021 / Accepted: December 23, 2021 / Published online: January 23, 2022

(C) The Author(s) 2022, corrected publication 2022

\section{ABSTRACT}

The ongoing coronavirus disease 2019 (COVID19) pandemic continues to raise questions for people living with multiple sclerosis (MS) and their healthcare providers. Common questions have included whether people living with MS are at higher risk of COVID-19 or of severe disease, whether certain disease-modifying therapies (DMTs) for MS heighten COVID-19 risk, and if/how COVID-19 vaccinations should be administered in relation to MS treatments. AntiCD20 therapies, which target B cells, have been of particular interest given the role B cells play in the response to both the virus that causes

Supplementary Information The online version contains supplementary material available at https:// doi.org/10.1007/s40120-021-00321-9.

R. K. Shin $(\square)$

MedStar Georgetown University Hospital, 3800 Reservoir Rd NW, Washington, DC 20007, USA

e-mail: Robert.K.Shin@gunet.georgetown.edu

P. Repovic

Multiple Sclerosis Center, Swedish Neuroscience

Institute, Seattle, WA, USA

e-mail: Pavle.Repovic@swedish.org

\section{J. R. Berger}

Perelman School of Medicine, University of

Pennsylvania, Philadelphia, PA, USA

e-mail: Joseph.Berger@pennmedicine.upenn.edu
COVID-19 (SARS-CoV-2) and vaccines. As more data surfaces and the pandemic evolves, additional questions have emerged regarding the administration of booster shots and differences between B cell-targeting therapies and other DMTs in terms of their immunomodulatory effects. In this podcast article, MS specialists discuss these challenges to MS care during the COVID-19 pandemic and the recent data which are currently informing their clinical decisionmaking. As the pandemic evolves, providers should continually partner with people living with MS to achieve MS treatment goals informed by the latest developments in COVID19.

Keywords: B-cell-targeting

therapy; Coronavirus; COVID-19; Disease modifying therapy; Multiple sclerosis; Boosters; SARSCoV-2; Vaccines 


\section{Key Summary Points}

The COVID-19 pandemic has led to many concerns for people living with multiple sclerosis (MS) and their providers, in part due to the immunomodulatory nature of MS disease-modifying therapies.

Recent studies suggest that MS itself is not a risk factor for COVID-19 and that disease-modifying therapies for MS likewise do not increase the risk of COVID-19.

People living with MS will benefit from COVID-19 vaccination, even if this benefit is lessened relative to those who are not on immunomodulatory/ immunosuppressive medications.

Providers should be aware of COVID-19 sequalae that may present similarly to MS.

\section{DIGITAL FEATURES}

This article is published with digital features, including a podcast audio file, to facilitate understanding of the article. To view digital features for this article go to https://doi.org/10. 6084/m9.figshare.17430008.

\section{PODCAST TRANSCRIPT}

\section{INTRODUCTION}

\section{Dr Shin:}

Welcome to our podcast, my name's Bob Shin. I'm a neurologist at MedStar Georgetown University hospital. I'm joined by two of my colleagues for this conversation.

Dr Berger:

I'm Joe Berger. I'm a professor of neurology at the University of Pennsylvania and associate chief for the Division of Multiple Sclerosis.
Dr Shin:

And we also have joining us Dr. Pavle Repovic.

Dr Repovic:

Hello. I am a neurologist in Seattle at the Multiple Sclerosis (MS) Center at the Swedish Neuroscience Institute. I also hold position of medical director for neurology research.

Dr Shin:

So, the three of us are going to have a conversation focusing on challenges in optimal MS care during this coronavirus disease 2019 (COVID-19) pandemic and then cover some best practices from our point of view.

\section{CHALLENGES TO OPTIMAL MS CARE DURING THE COVID-19 PANDEMIC}

Dr Shin:

There have been a lot of concerns surrounding COVID-19, just from a medical standpoint, and certainly within the MS space. We can just start the conversation with what we're thinking in terms of these unique concerns.

Dr Berger:

There are actually, in my mind three concerns relevant to the MS community.

One is, what does COVID-19 do to the course of MS? Is COVID-19 going to increase your risk of having a relapse? We know from data that had been generated years ago by Bill Sibley that viruses in general seem to be a risk for increased relapse of this disease [1]. But curiously, we've not seen this really with COVID-19; we've seen pseudo-relapses, but relapses don't seem to be terribly much more prevalent in people who get infected, who are MS patients, than you'd expect otherwise.

Number two is, what does MS and MS treatments do to the course of COVID.

And then question number three is more for the MS community of physicians. And that is, are there illnesses that look like MS that are triggered by COVID-19? And it turns out there are.

We're going to focus largely on the effects of MS and, more importantly, disease-modifying therapies (DMTs) on the course of COVID-19. 
But I think you shouldn't forget about these others as well.

Dr Shin:

I think we're in agreement that it doesn't look like people living with MS are intrinsically more vulnerable to COVID-19 infection. I think we can agree on that?

Dr Berger:

That's correct. That's been looked at, and the people with MS at increased risk are those individuals who have those same risk factors as individuals without MS. In other words, obesity, metabolic syndrome, diabetes, lung disease, cardiac disease, age, being a male. Those factors increase one's risk, whether you have MS or you don't have MS [2-4].

Dr Shin:

What about, let's say, our treatments for multiple sclerosis-they're immunomodulatory, immunosuppressant. From your point of view, Dr Repovic, how should we be factoring this into our conversation in COVID-19 and MS?

Dr Repovic:

So specifically with regard to the risk of COVID, I'm aware of one study that looked at the health claims data, namely Tony Reder's study with about 25,000 insurance claims, where they were actually able to draw some conclusions about different disease-modifying therapies and the risk of being diagnosed with COVID [5]. For most MS therapies, that risk was sort of at the base, at the background level, if you will. There were no differentiators for injectable therapies, interferons, and glatiramer. I think for some of the B-cell therapies, CD20targeting therapies, there was an increased risk. So, from that study, which is sizeable, one might conclude that there's perhaps an increased risk, but it's always important to remember that these sorts of studies carry potential biases.

Dr Repovic:

And some of which may not be really accounted for, such as age, behavior patterns, and also vaccination status. As far as the risk of more severe COVID, I have to say that the data that I look at for this is largely derived from national COVID databases, including the one in the USA, and Italy, France, Sweden, Spain, perhaps. So a combination of these, or other collaborative efforts between these and some others recently published in Neurology, suggest that again, for most MS therapies, there is no increased risk of being hospitalized.

Dr Repovic:

That's a surrogate of severity of COVID or being admitted to the intensive care unit. But the exception to that rule, or rather to that trend, was seen with patients who are on ocrelizumab and rituximab-with more of those patients having an increased risk of being hospitalized [4]. So I would say that this suggests that, at least for those populations, there might be an increased risk of being hospitalized.

Dr Shin:

I always think it's interesting. You brought up the concern that there's something about B-cell therapies that may either increase risk of infection or predispose to more severe infection.

Before we talk about that a little more, I think we should take just a moment to sketch out current thinking about immune responses in general? Dr. Berger, if we're just giving a very simple outline... What is the immune response?

Dr Berger:

I'm happy to do that. Recall that when you are exposed to a novel antigen and for almost all of us, the SARS-CoV-2 virus causing COVID19 is novel. The component of the immune system that deals with it is the innate immune system. And that can be quite a considerable immune response and protect you in a way that you never ever develop significant infection, allowing time for the adaptive immune system to kick in.

Dr Berger:

And what happens with the adaptive immune system? There are two components to it. One is humoral immunity where the plasma cells start generating antibodies. And the other is the cell-mediated response. Now the antibodies that you generate (and these are generated after vaccination-both that and the cellmediated response), the antibodies that you generate bind to the spike protein of SARS-CoV2 , and prevent that virus from adhering to angiotensin-converting receptor 2 , that is found on certain cells. This binding actually prevents 
infection; you have high antibody levels, it may prevent infection $[6,7]$.

Dr Berger:

The cell-mediated response that you have is probably more important because of what it does: it gets into the infected tissues and clears out the virus from those infected tissues.

Dr Berger:

We have a large number of patients on B-cell therapies in our practice. And we really haven't seen that they have a higher risk of severe disease as evidenced by increased hospitalization or death. On the other hand, I am aware of what's come out of the registries. And what it appears is that these patients have a slightly increased risk over the rest of the MS population of hospitalization-not of death, per se-but of hospitalization. And, this sort of makes sense because the humoral responses we know from a number of studies can be abrogated by the administration of a B-cell depleter. On the other hand, at least some of the studies have demonstrated that the $\mathrm{T}$ cell-mediated response is unaffected [8].

Dr Berger:

And if it is affected, it seems to be more robust in these patients who have been treated with drugs like ocrelizumab than seen in a control population [8]. So while you may have lowered the humoral response, the cell-mediated response may be more robust than you would otherwise see. I think by and large, it's something that we should be concerned about, but not overly concerned about.

Dr Berger:

Sir William Osler, who was a worldrenowned internist, said, "Medicine is a science of uncertainty, and an art of probability." We certainly have to apply that when it comes to COVID, we just don't know enough to make real strong recommendations, in my opinion.

Dr Shin:

And I think all of us have had patients who have come to us say, "Oh, should I get my COVID-19 antibody levels checked? Do I have protection? How do you counsel your patients or, what is your take on those observations?

Dr Repovic:

So the topic of testing antibody titers to SARS-CoV-2 in MS patients who have been vaccinated, for the purpose of determining the efficacy of that vaccination is pretty fraught, I would say, with pitfalls. So I definitely don't recommend testing for antibodies to SARS-CoV2 in all of the patients; that said, when a person is particularly nervous about their own plans to return to work for example. That's a situation that I recently had to deal with where an individual was facing a request by their employer to return to their place of employment, where there isn't a vaccine mandate and masking is not particularly strictly enforced. We had to make a decision what to do in this situation.

Dr Repovic:

Now the data so far from the anti-SARS-CoV2 investigations suggest that somewhere around $20-30 \%$ of people on ocrelizumab or rituximab generate antibodies, but the majority don't $[9,10]$. So there is a possibility that a person might have antibodies, or not. And you could use this uncertainty perhaps to justify to the employer why this individual should perhaps be allowed to work from home for another period of time.

Dr Repovic:

Again, it's not a perfect situation. And I fully recognize that this is not something that everybody will agree with, but for us in that sort of situation, it did make sense.

Dr Shin:

I have to admit that in my own practice I strongly discourage my patients from getting antibody testing in this context. I did want to throw one more question in this area to you, which is, we've been focusing a lot on B-cell therapies. Do you think the evidence suggests that B-cell therapies are all essentially created equal? Is it just that all anti-CD20 agents basically have the same effect or have we seen differences comparing the different anti-CD20 agents?

Dr Repovic:

They certainly have similar mechanism of action, but the data, at least the data we've referenced or alluded to so far, would actually not point out that they're all interchangeable. So from these national registries, the risk with rituximab appears to be somewhat higher than that with ocrelizumab and with ofatumumab $[4,11]$. So I think there may still be some shades 
of differentiation between them in terms of risk, and in terms of immune compatibility with vaccines.

Dr Berger:

I think that although the studies are still preliminary, it appears that the ability to generate antibodies with ofatumumab as opposed to ocrelizumab is somewhat higher. So a higher percentage of individuals who are treated with ofatumumab and who get the vaccine seem to generate a measurable antibody response $[9,10,12]$.

\section{VACCINATION}

Dr Shin:

Well, I do think it is interesting that, as you hinted, it's early, and we're just really learning about this. But there have been a number of reports that have suggested that the ability to see an antibody response might be higher in patients who had received ofatumumab versus some of the others-let's say the infused antiCD20 agents. And we're starting to see differences in the likelihood that one might develop hypo-IgG, for example [11, 13-20]. Again, this may or may not really be relevant in this context, but there is this idea that maybe they're not all created equal.

Dr Shin:

I did want to shift the conversation; I do think we need to talk about vaccination. We keep getting different recommendations from different agencies on who should be getting vaccinated, when you should be getting vaccinated, should you be getting a booster. But if we focus in specifically on MS patients, how have you been counseling your patients in terms of should they be getting the COVID-19 vaccine? If so, are there special considerations regarding timing?

Dr Repovic:

So I absolutely think MS patients should be vaccinated against COVID [21, 22]. I think there was certainly a lot of concern initially among patients and clinicians regarding how these vaccines would play out and how safe they would be, and so forth. I would probably say that our own personal experience with vaccines over this past year has supported the notion that the relapses of MS do not appear to be more common following vaccination.

Dr Repovic:

And so I do recommend vaccination against COVID to all of my patients, whether or not they've had COVID earlier. And regardless of which disease-modifying therapy they're on, because you know, any protection is better than no protection.

Dr Shin:

I think it's great. I still occasionally encounter resistance. People will say, "Well, they haven't studied the vaccine specifically in people with multiple sclerosis, or we don't have enough time." But I feel like our message should be quite firm. Everyone needs to get vaccinated. Everybody with multiple sclerosis needs to get vaccinated. But, Dr. Berger, what do you think about the recommendations on timing that have been put out there?

Dr Berger:

I'd agree with Pavle, and with you, that every patient needs to be vaccinated. Now where it becomes an issue is the timing in individuals on infusion B-cell depleters, because what's been demonstrated, starting with the VELOCE trial, is that in individuals who receive vaccines following infusions, anti-CD20 monoclonal antibodies do not mount the same antibody response as in individuals who are on other disease-modifying therapies [23]. So the question then becomes, what's the ideal timing following the 6-month infusions?

Dr Berger:

And the recommendation has been is wait at least 3 months after the infusion, preferably 4 months, but not to get too close to the next infusion because that'll attenuate the response [24]. So if you have a vaccine, if it's the initial series of vaccine and if it's an mRNA vaccine, and you're giving it at 21 days or 28 days, you've got to time it so that that second vaccine is given at least 1 month before the infusion occurs. That would be ideal. The problem is that life isn't like that. I mean, it's much messier.

So I have what I call my potential patient scenarios. Let's talk about the unvaccinated population first. So there are patients who come into your office who have not been on a disease- 
modifying therapy, who you want to start on an anti-CD20, and they've not been vaccinated. Some of those patients may have a disease like primary progressive MS.

Dr Berger:

I think that it's quite acceptable to hold off on the infusions until you have that person fully vaccinated. That makes sense to me.

On the other hand, you'll have patients with rip-roaring relapsing-remitting MS. And you say to yourself, "Look, I got to get this disease under control." And the disease is much more important to you than the COVID-19 at that time, because the latter is only a possibility. So those people, I start on the drug. And in fact, in terms of vaccinating them, depending on where they live, if they're in an area of high-risk COVID-19, I just say, go ahead and get vaccinated.

\section{Dr Berger:}

Then you have those individuals who have already been vaccinated. And you're now talking about administering a booster and, in those instances, I think you have a lot more latitude because they've already been vaccinated. So in those individuals, I've been saying, "You know what? Wait a minimum of 3 months after your infusion. And we'll give you the booster then." With the exception of individuals who live in high-risk COVID-19 areas. I might want to get them boostered sooner, but again, it's the art of medicine, not the science of medicine.

Dr Shin:

Sure. Pavle, do you practice in a similar way?

Dr Repovic:

Definitely not sticking to these timelines as some sort of a rigid guideline. So the initial vaccination series earlier this year, of course, everybody who wants to get vaccinated, wanted to get vaccinated yesterday. I wasn't going to stand in their way and insist on a 3-month waiting period. But now with the boosters, what we are doing, actually, we're looking at a schedule of people who are scheduled for infusion in 1-2 months from now. And we're contacting them and asking them if they've gotten boosters.

Dr Repovic:

It has generated that sort of a discussion of trying to find the optimal time and how to communicate that to a large number of patients who remain on these therapies so they can take advantage of that window, presumably the optimal window of opportunity.

Dr Shin:

It does sound like we all had a very similar approach. The priority, at least initially, was for our patients to get vaccinated. Trying to be sensitive to time may have been a factor, but the priority was to get them vaccinated. But I actually $100 \%$ agree with both of you that when it comes to the booster concept, I think we do have the luxury of time.

\section{FUTURE PERSPECTIVES}

Dr Shin:

I did want to leave some time. Dr. Berger, you had your three points that you wanted to bring up. Maybe you can flesh it out, what you were hinting at, that COVID-19 infection does appear to be associated with a variety of different post-infectious complications.

Dr Berger:

We do know that illnesses like acute disseminated encephalomyelitis [25], optic neuritis [26], neuromyelitis optica (NMO), neuromyelitis optica spectrum disorder (NMOSD) [27], transverse myelitis [28], that all of these, which can look like MS, have been reported in the face of acute COVID-19.

Dr Berger:

To me, it's not surprising because we see this with other viral illnesses as well. It's not unique to COVID-19. And in fact, the frequency with which it occurs is not very high. It's just that some of these presentations can be mistaken for multiple sclerosis, and physicians need to be aware that there are autoimmune diseases that can be triggered by COVID-19. Interestingly, we tend to conceptualize these complications as occurring 2-3 weeks after the infection. But with COVID-19, most of the reports suggest that they occur concurrently. It's not that it can't occur afterwards, some do. But while you have acute infection, you may develop your optic neuritis or your acute disseminated encephalomyelitis.

Dr Shin: 
Well, I guess in the MS world, we're increasingly being vigilant for things like aquaporin 4 antibodies and MOG antibodies [29]. And so I think certainly when we're seeing something that looks like MS evolving after COVID-19 infection, I'm definitely wondering if it's another type of auto-antibody.

Dr Berger:

So I will say that both have been reported, case reports following COVID-19, that is aquaporin 4 positivity with NMO [30] and anti-MOG with the NMOSD [31].

Dr Repovic:

Just one other comment related to sequelae of COVID that might look like MS. We're still not entirely sure about the prevalence of long COVID symptoms. So there's those with protracted recovery, and the question of whether that's more likely to occur in patients with multiple sclerosis. The large UK-based study suggested that individuals with greater disability, with preexisting anxiety, and of female sex tend to have actually higher odds of developing long COVID—this relates specifically to MS patients [32]. So I think that I often reference that when I discuss the rationale for COVID vaccination with my vaccine hesitant patients because they really do need to understand that their odds of some of these outcomes are not always trivial.

Dr Shin:

Pavle, you had hinted at the idea of treatment of COVID-19 with certain kinds of antibody therapies. Did you want to give us any thoughts on that approach?

Dr Repovic:

So, as far as the treatment options against COVID specifically with our MS disease-modifying therapies, I've welcomed the US Food and Drug Administration emergency use authorization for the use of monoclonal antibodies in people who are on medications that can interfere with vaccine efficacy.

Dr Repovic:

I routinely make it a point of letting my patients on B-cell therapies and S1Ps know that if they should become infected with COVID, they should seek this treatment.

\section{CONCLUDING REMARKS}

\section{Dr Shin:}

So I did want to try to summarize things a little bit. We started talking about the risk of COVID-19 to or the expression of COVID-19 infection in individuals with MS. We agree that, for the most part, our patients with MS are not at increased risk from the infection, and that this, for the most part, is regardless of what disease-modifying therapy they're on. We did spend a lot of time focusing on anti-CD20 agents in particular, based on certain observations that might have raised a question of whether there is less immunity, but it seems that as we're still using the anti-CD20 agents, we still believe that the risk-benefit profile tilts in favor of using it for the appropriate patients.

Dr Shin:

We also spent a lot of time talking about vaccination issues, and I think we agree that people living with MS benefit from vaccination, really, regardless of which therapy they're on. And, Pavle, you mentioned that we need to just make sure that they're aware that if they were to have the symptoms of COVID, they should seek attention as soon as possible, in order to access treatments like monoclonal antibody therapies.

I want to give each of you an opportunity to give some last thoughts or any other reflections that you might want to have on how things have changed in our practice during this pandemic.

Dr Berger:

Well, things have changed tremendously since we began seeing patients via telemedicine, something that was not available to us before the pandemic. I do think that some patients experience seeing their doctors via telemedicine as a tremendous convenience, particularly those who find it very difficult to get into the clinic and/or who have to travel long distances, or those that have physical disabilities. I will add the following, and this will be my concluding remarks before I turn it over to Pavle.

Dr Berger:

I think that SARS-CoV-2, the virus, is going to be an endemic virus, and we'll just simply get accustomed to it and we'll make 
accommodations for it. And we'll have better treatments come out with drugs other than antibodies.

Dr Berger:

In a year's time, if the three of us get together, there's every possibility we're going to say something quite different from what we said tonight. And it reminds me of what Ralph Waldo Emerson said, which is, "Foolish consistency is the hobgoblin of small minds." So, I would think that as we learn more, we're just going to modify our opinions.

Dr Shin:

All right. Dr. Repovic, any final thoughts or reflections?

Dr Repovic:

Our responsibility to our patients remains to treat their MS in the best, most effective and most reasonable way. We may all get really wrapped up, twisted in a pretzel over the vaccine, over the COVID, over telemedicine, over everything. But at the end of the day, we truly have to keep the eye on the goal, which is to treat this disease as best we possibly can. So sacrificing effective therapies because they carry certain risks is literally shooting ourselves in the foot. But being mindful of those risks is the first step to reducing them, recognizing who is at a higher risk, figuring out how to mitigate those risks, either through the use of preventative monoclonal antibodies (following the recording of this podcast, monoclonal antibody therapy for pre-exposure prophylaxis was granted an Emergency Use Authorization by the FDA [33]) or some other prophylactic methods or something else is our next challenge, because I agree with Dr. Berger, that the virus is here to stay.

Dr Shin:

I really appreciate the opportunity to spend time with the two of you. I actually learned a lot in this, during this podcast, from you. Thank you both.

\section{ACKNOWLEDGEMENTS}

Funding. The journal's Rapid Service Fee was funded by Novartis Pharmaceuticals
Corporation. No funding was provided for the authoring of this review. The authors received no honoraria related to the development of this publication.

Medical Writing. Medical writing support was funded by Novartis Pharmaceuticals Corporation. This medical writing support, including assisting authors with the development of the manuscript drafts and incorporation of comments, was provided by Meredith Whitaker, PhD, of Alphabet Health (New York, NY), according to Good Publication Practice guidelines (https://www.ismpp.org/gpp3).

Authorship. All named authors meet the International Committee of Medical Journal Editors (ICMJE) criteria for authorship for this article, take responsibility for the integrity of the work as a whole, and have given their approval for this version to be published.

Authors' Contributions. All authors made substantial contributions to the manuscript concept/design, critically reviewed and revised the manuscript drafts, and provided final approval of the manuscript and enhanced content as submitted.

Disclosures. The views expressed in the following podcast article content are the opinion and perspective of the medical expert authors. Opinions expressed in this article do not necessarily reflect those of Novartis, and local labels, guidance, etc. should be followed. Pavle Repovic has received consulting and/or speaking honoraria from Alexion, Biogen, Celgene, Roche, Sanofi Genzyme, Viela, and EMD Serono. Joseph R Berger has received grant support from Biogen and Genentech, and consultant fees from Amgen, Biogen, Celgene, Encycle, Excision-Bio, Genentech/Roche, Genzyme, Inhibikase, Millennium/Takeda, Novartis, Sanofi/Genzyme, Shire, and Serono. Robert K Shin has received personal compensation as a consultant or speaker for Biogen, Bristol Myers Squibb, EMD Serono, Genentech, Mallinckrodt, Novartis, and Sanofi Genzyme. 
Compliance with Ethics Guidelines. This article does not contain any studies with human participants or animals performed by any of the authors.

Open Access. This article is licensed under a Creative Commons Attribution-NonCommercial 4.0 International License, which permits any non-commercial use, sharing, adaptation, distribution and reproduction in any medium or format, as long as you give appropriate credit to the original author(s) and the source, provide a link to the Creative Commons licence, and indicate if changes were made. The images or other third party material in this article are included in the article's Creative Commons licence, unless indicated otherwise in a credit line to the material. If material is not included in the article's Creative Commons licence and your intended use is not permitted by statutory regulation or exceeds the permitted use, you will need to obtain permission directly from the copyright holder. To view a copy of this licence, visit http://creativecommons.org/licenses/by$\mathrm{nc} / 4.0 /$.

\section{REFERENCES}

1. Sibley WA, Bamford CR, Clark K. Clinical viral infections and multiple sclerosis. Lancet. 1985;1(8441):1313-5.

2. Chaudhry F, Jageka C, Levy PD, Cerghet M, Lisak RP. Review of the COVID-19 risk in multiple sclerosis. J Cell Immunol. 2021;3(2):68-77.

3. National Multiple Sclerosis Society. Multiple sclerosis and COVID-19. 2021. https://www.national mssociety.org/coronavirus-covid-19-information/ multiple-sclerosis-and-coronavirus\#section-0. Accessed 6 Oct 2021

4. Salter A, Fox RJ, Newsome SD, et al. Outcomes and risk factors associated with SARS-CoV-2 infection in a North American Registry of patients with multiple sclerosis. JAMA Neurol. 2021;78(6):699-708.

5. Reder AT, Centonze D, Naylor ML, et al. COVID-19 in patients with multiple sclerosis: associations with disease-modifying therapies. CNS Drugs. 2021;35(3):317-30.
6. Centers for Disease Control and Prevention. Understanding how COVID-19 vaccines work. 2021. https://www.cdc.gov/coronavirus/2019-ncov/ vaccines/different-vaccines/how-they-work.html. Accessed 28 May 2021.

7. Pollard AJ, Bijker EM. A guide to vaccinology: from basic principles to new developments. Nat Rev Immunol. 2021;21(2):83-100.

8. Apostolidis SA, Kakara M, Painter MM, et al. Cellular and humoral immune responses following SARS-CoV-2 mRNA vaccination in patients with multiple sclerosis on anti-CD20 therapy. Nat Med. 2021;27:1990-2001.

9. Karussis D, Karni A, Milo R, Staun-Ram E, Miller A. Humoral immune response to COVID-19 mRNA (BNT162b2-Pfizer) vaccine in patients with multiple sclerosis. In: European Committee for Treatment and Research in Multiple Sclerosis (ECTRIMS) 2021. Virtual Meeting. 2021. https://ectrims2021.abstract server.com/program/\#/details/presentations/1510. Accessed 16 Nov 2021.

10. Oreja-Guevara C, Ramirez CI, Alba-Suarez E, et al. COVID-19 vaccination and humoral immune response in people with multiple sclerosis. In: 37 th Congress of the European Committee for the Treatment and Research in Multiple Sclerosis (ECTRiMS). 2021. https://doi.org/10.1177/ 13524585211044667. Accessed 1 Nov 2021.

11. Simpson-Yap S, De Brouwer E, Kalincik T, et al. Associations of disease-modifying therapies with COVID-19 severity in multiple sclerosis. Neurology. 2021;97(19):e1870-85.

12. Noh H, Mortensen T, Dave RH. COVID-19 vaccine antigenicity in the setting of MS disease modifying treatments. In: Consortium of Multiple Sclerosis Centers (CMSC) 2021 annual meeting, 25-28 Oct 2021, Orlando. https://2021abstracts.cmscscholar. org/2021/11/15/covid-19-vaccine-antigenicity-inthe-setting-of-ms-disease-modifying-treatments/. Accessed 4 Jan 2021.

13. Genentech, Inc. RITUXAN ${ }^{\circledR}$ (rituximab) [package insert]. https://www.gene.com/download/pdf/ rituxan_prescribing.pdf. Accessed 29 Nov 2021.

14. U.S. Food and Drug Administration. OCREVUS ${ }^{\circledR}$ (ocrelizumab) [package insert]. 2021. https://www. accessdata.fda.gov/drugsatfda_docs/label/2016/125 326s062lbl.pdf. Accessed 29 Nov 2021.

15. Bar-Or A, Calabresi PA, Arnold D, et al. Rituximab in relapsing-remitting multiple sclerosis: a 72-week, open-label, phase I trial. Ann Neurol. 2008;63(3): 395-400. 
16. Hauser SL, Bar-Or A, Comi G, et al. Ocrelizumab versus interferon beta-1a in relapsing multiple sclerosis. N Engl J Med. 2017;376(3):221-34.

17. Hauser SL, Kappos L, Arnold DL, et al. Five years of ocrelizumab in relapsing multiple sclerosis: OPERA studies open-label extension. Neurology. 2020;95(13):e1854-67.

18. Hauser SL, Waubant E, Arnold DL, et al. B-cell depletion with rituximab in relapsing-remitting multiple sclerosis. N Engl J Med. 2008;358(7): 676-88.

19. Wiendl H, de Sèze J, Bar-Or A, et al. Effect of ofatumumab on serum immunoglobulin levels and infection risk in patients with relapsing multiple sclerosis over 3.5 years [ECTRIMS 2021-Late Breaking News ePoster]. Mult Scler J. 2021; 27(2_suppl):752-804.

20. Tsao L, Otani IM, Bove R. Hypogammaglobulinemia in multiple sclerosis patients receiving diseasemodifying immunomodulatory agents. J Allergy Clin Immunol. 2019;143(2):AB16.

21. National Multiple Sclerosis Society. COVID-19 vaccine guidance for people living with MS. 2021. https://www.nationalmssociety.org/coronaviruscovid-19-information/multiple-sclerosis-and-corona virus/covid-19-vaccine-guidance. Accessed 24 Nov 2021.

22. Shin RK, Rammohan KW, Williams MJ. Expert perspectives on COVID-19 vaccination for people living with multiple sclerosis. Neurol Ther. $2021 ; 10(2): 415-25$.

23. Bar-Or A, Calkwood JC, Chognot C, et al. Effect of ocrelizumab on vaccine responses in patients with multiple sclerosis: the VELOCE study. Neurology. 2020;95(14):e1999-2008.

24. National Multiple Sclerosis Society. Timing MS medications with COVID-19 vaccines. 2021. https://www.nationalmssociety.org/coronaviruscovid-19-information/multiple-sclerosis-and-corona virus/covid-19-vaccine-guidance/Timing-MS-Medica tions-with-COVID-19-Vaccines. Accessed 24 Nov 2021.

25. Langley L, Zeicu C, Whitton L, Pauls M. Acute disseminated encephalomyelitis (ADEM) associated with COVID-19. BMJ Case Rep. 2020;13(12): e239597.

26. Azab MA, Hasaneen SF, Hanifa H, Azzam AY. Optic neuritis post-COVID-19 infection. A case report with meta-analysis. Interdiscip Neurosurg. 2021;26: 101320.

27. de Ruijter NS, Kramer G, Gons RAR, Hengstman GJD. Neuromyelitis optica spectrum disorder after presumed coronavirus (COVID-19) infection: a case report. Mult Scler Relat Disord. 2020;46:102474-4.

28. Román GC, Gracia F, Torres A, Palacios A, Gracia K. Harris D Acute transverse myelitis (ATM): clinical review of 43 patients with COVID-19-associated ATM and 3 post-vaccination ATM serious adverse events with the ChAdOx1 nCoV-19 vaccine (AZD1222). Front Immunol. 2021;12:653786.

29. National Multiple Sclerosis Society. Neuromyelitis Optica (NMO). 2019. https://www.nationalmssociety. org/What-is-MS/Related-Conditions/NeuromyelitisOptica-(NMO). Accessed 29 Nov 2021.

30. Corrêa DG, de Souza Lima FC, da Cruz Bezerra D, Coutinho AC. Hygino da Cruz LC COVID-19 associated with encephalomyeloradiculitis and positive anti-aquaporin- 4 antibodies: cause or coincidence? Mult Scler J. 2021;27(6):973-6.

31. Sardar S, Safan A, Okar L, Sadik N. Adeli G The diagnostic dilemma of bilateral optic neuritis and idiopathic intracranial hypertension coexistence in a patient with recent COVID-19 infection. Clin Case Rep. 2021;9(6):e04347.

32. Garjani A, Middleton RM, Nicholas R, Evangelou N. Pre-existing anxiety, depression, and neurological disability is associated with long COVID: A prospective and longitudinal cohort of the United Kingdom Multiple Sclerosis Register. medRxiv. 2021. https://doi.org/10.1101/2021.06.25.2125 9256.

33. US Food and Drug Administration. Coronavirus (COVID-19) Update: FDA authorizes new long-acting monoclonal antibodies for pre-exposure prevention of COVID-19 in certain individuals. 2021. https:// www.fda.gov/news-events/press-announcements/ coronavirus-covid-19-update-fda-authorizes-newlong-acting-monoclonal-antibodies-pre-exposure. Accessed 19 Dec 2021. 